\title{
VLT multi-object spectroscopy of 33 eclipsing binaries in the Small Magellanic Cloud
}

\section{New distance and depth of the SMC, and a record-breaking apsidal motion (Corrigendum)}

\author{
P. North ${ }^{1}$, R. Gauderon ${ }^{1}$, F. Barblan ${ }^{2}$, and F. Royer ${ }^{3}$ \\ ${ }^{1}$ Laboratoire d'Astrophysique, École Polytechnique Fédérale de Lausanne (EPFL), Observatoire, 1290 Versoix, Switzerland \\ e-mail: pierre.north@epfl.ch \\ 2 Geneva Observatory, Geneva University, 1290 Sauverny, Switzerland \\ e-mail: fabio.barblan@unige.ch \\ 3 GEPI, UMR 8111 du CNRS, Observatoire de Paris-Meudon, 92195 Meudon Cedex, France \\ e-mail: frederic.royer@obspm.fr
}

A\&A 520, A74 (2010), DOI: 110.1051/0004-6361/200810284

Key words. binaries: eclipsing - binaries: spectroscopic - stars: early type - Magellanic Clouds - stars: fundamental parameters Errata, addenda

Minus signs had been accidentally dropped in Table 9, which compares the dynamical mass with the theoretical mass obtained from interpolation of evolutionary tracks in the HR diagram. Furthermore, the error bars had been omitted. We provide here the corrected Table 9 . The errors on $\Delta \mathcal{M}$ are computed according to the formula

$\sigma^{2}(\Delta \mathcal{M})=\sigma^{2}(\mathcal{M})+\sigma^{2}\left(\mathcal{M}_{\text {int }}\right)$

where $\mathcal{M}$ is the dynamical mass and $\mathcal{M}_{\text {int }}$ is the mass interpolated in the HR diagram from the theoretical evolutionary tracks. One has

$\sigma\left(\mathcal{M}_{\text {int }}\right)=\frac{1}{2}\left[\mathcal{M}_{\text {int }}\left(T_{\text {eff,max }}, L_{\text {max }}\right)-\mathcal{M}_{\text {int }}\left(T_{\text {eff,min }}, L_{\text {min }}\right)\right]$

with $T_{\text {eff,max,min }}=T_{\text {eff }} \pm \sigma\left(T_{\text {eff }}\right)$ and $L_{\text {max, } \min }=L \pm \sigma(L)$.

The interpolation procedure was tested by running it on a grid of only 5 evolutionary tracks $\left(3,5,9,15,25 M_{\odot}\right)$ instead of $9\left(2.5,3,4,5,7,9,12,15,20 M_{\odot}\right)$; it recovered the values interpolated in the full grid to better than $0.01 M_{\odot}$ in most cases, an to within a few times $0.01 M_{\odot}$ in some unfavourable cases.

Figure 9, which corresponds to Table 9, was correct.

These corrections do not affect the conclusion that many components of detached systems appear slightly overluminous for their dynamical mass, or that their evolutionary mass tend to be larger than their dynamical mass, a trend also pointed out recently by Massey et al. (2012).

\section{References}

Massey, P., Morrell, N. I., Neugent, K. F., et al. 2012, ApJ, in press [arXiv: astro-ph/1201.3280]
Table 9. Comparison with theoretical evolutionary models: difference between the evolutionary and observed masses.

\begin{tabular}{lcrr}
\hline \hline Object & Model & \multicolumn{1}{c}{$\Delta \mathcal{M}_{\mathrm{P}}$} & \multicolumn{1}{c}{$\Delta \mathcal{M}_{\mathrm{S}}$} \\
& & \multicolumn{1}{c}{$\left(\mathcal{M}_{\odot}\right)$} & \multicolumn{1}{c}{$\left.\mathcal{M}_{\odot}\right)$} \\
\hline 4110409 & $\mathrm{sd}$ & $1.08 \pm 0.99$ & $1.92 \pm 0.55$ \\
4113853 & $\mathrm{sd}$ & $1.50 \pm 0.58$ & $0.85 \pm 0.39$ \\
4117831 & $\mathrm{~d}$ & $0.39 \pm 0.31$ & $0.37 \pm 0.35$ \\
4121084 & $\mathrm{~d}$ & $1.50 \pm 0.65$ & $1.40 \pm 0.58$ \\
4121110 & $\mathrm{~d}$ & $1.48 \pm 0.64$ & $0.92 \pm 0.47$ \\
4121461 & $\mathrm{~d}$ & $0.73 \pm 0.47$ & $0.43 \pm 0.50$ \\
4159928 & $\mathrm{sd}$ & $1.09 \pm 0.84$ & $0.57 \pm 0.46$ \\
4160094 & $\mathrm{~d}$ & $0.85 \pm 0.94$ & $0.26 \pm 0.71$ \\
4163552 & $\mathrm{~d}$ & $-0.22 \pm 0.86$ & $-0.20 \pm 0.83$ \\
4175149 & $\mathrm{sd}$ & $4.14 \pm 0.60$ & $5.09 \pm 0.46$ \\
4175333 & $\mathrm{~d}$ & $1.01 \pm 0.37$ & $0.80 \pm 0.32$ \\
5016658 & $\mathrm{~d}$ & $0.30 \pm 0.48$ & $0.65 \pm 0.45$ \\
5026631 & $\mathrm{sd}$ & $0.92 \pm 0.47$ & $-0.46 \pm 0.32$ \\
5032412 & $\mathrm{~d}$ & $0.92 \pm 0.44$ & $0.21 \pm 0.42$ \\
5038089 & $\mathrm{~d}$ & $1.76 \pm 0.26$ & $2.35 \pm 0.34$ \\
5095337 & $\mathrm{~d}$ & $1.24 \pm 0.90$ & $0.93 \pm 0.76$ \\
5095557 & $\mathrm{~d}$ & $1.22 \pm 0.75$ & $0.64 \pm 0.68$ \\
5100485 & $\mathrm{~d}$ & $0.41 \pm 0.30$ & $0.63 \pm 0.31$ \\
5100731 & $\mathrm{c}$ & $-0.25 \pm 0.61$ & $-0.69 \pm 0.60$ \\
5106039 & $\mathrm{sd}$ & $0.81 \pm 0.44$ & $1.12 \pm 0.26$ \\
5111649 & $\mathrm{~d}$ & $0.16 \pm 0.19$ & $0.48 \pm 0.20$ \\
5123390 & $\mathrm{~d}$ & $1.24 \pm 0.51$ & $2.57 \pm 0.60$ \\
5180185 & $\mathrm{~d}$ & $-0.39 \pm 0.24$ & $0.05 \pm 0.33$ \\
5180576 & $\mathrm{~d}$ & $1.12 \pm 0.70$ & $-0.08 \pm 0.55$ \\
5185408 & $\mathrm{~d}$ & $0.77 \pm 0.35$ & $0.94 \pm 0.38$ \\
5196565 & $\mathrm{~d}$ & $0.45 \pm 0.36$ & $-0.24 \pm 0.32$ \\
5261267 & $\mathrm{sd}$ & $1.92 \pm 0.77$ & $1.57 \pm 0.39$ \\
5265970 & $\mathrm{~d}$ & $0.00 \pm 0.43$ & $0.26 \pm 0.36$ \\
5266015 & $\mathrm{sd}$ & $0.39 \pm 0.62$ & $2.74 \pm 0.32$ \\
5266131 & $\mathrm{~d}$ & $0.64 \pm 0.57$ & $0.45 \pm 0.49$ \\
5266513 & $\mathrm{~d}$ & $0.19 \pm 0.65$ & $0.46 \pm 0.58$ \\
5277080 & $\mathrm{sd}$ & $3.14 \pm 0.68$ & $2.33 \pm 0.35$ \\
5283079 & $\mathrm{~d}$ & $0.70 \pm 0.50$ & $0.67 \pm 0.52$ \\
\hline
\end{tabular}

Notes. The "P" and "S" subscripts stand for "primary" and "secondary" component respectively. 